\title{
On the Korovkin approximation theorem and Volkov-type theorems
}

Nihan Uygun ${ }^{*}$

\section{*Correspondence:} nuygun@ibu.edu.tr Department of Mathematics, Abant İzzet Baysal University, Gölköy Kampüsü, Bolu, 14280, Turkey

\begin{abstract}
In this short paper, we give a generalization of the classical Korovkin approximation theorem (Korovkin in Linear Operators and Approximation Theory, 1960), Volkov-type theorems (Volkov in Dokl. Akad. Nauk SSSR 115:17-19, 1957), and a recent result of (Taşdelen and Erençin in J. Math. Anal. Appl. 331(1):727-735, 2007).
\end{abstract}

MSC: Primary 41A36; 41A25

Keywords: positive linear operator; Korovkin theorem; Volkov-type theorem; modules of continuity; compact Hausdorff spaces

\section{Introduction}

In this paper, the classical Korovkin theorem (see [1]) and one of the key results (Theorem 1) of [2] will be generalized to arbitrary compact Hausdorff spaces. For a topological space $X$, the space of real-valued continuous functions on $X$, as usual, will be denoted by $C(X)$. We note that if $X$ is a compact Hausdorff space, then $C(X)$ is a Banach space under pointwise algebraic operations and under the norm

$$
\|f\|=\sup _{x \in X}|f(x)| .
$$

Let $X$ be a compact Hausdorff space and $E$ be a subspace of $C(X)$. Then a linear map $A: E \rightarrow C(X)$ is called positive if $A(f) \geq \mathbf{0}$ in $C(X)$ whenever $f \geq 0$ in $E$. Here $f \geq \mathbf{0}$ means that $f(x) \geq 0$ in $\mathbb{R}$ for all $x \in X$.

For more details on abstract Korovkin approximations theory, we refer to [3] and [4].

Constant-one function on a topological space $X$ will be denoted by $f_{0}$, that is, $f_{0}(x)=1$ for all $x \in X$. If $A=(a, b)$ and $B=(c, d)$ are elements of $\mathbb{R}^{2}$, then the Euclidean distance between $A$ and $B$, given by

$$
|(a, b)-(c, d)|=\sqrt{(a-c)^{2}+(b-d)^{2}},
$$

is denoted by $|A-B|$.

Definition 1.1 Let $X$ and $Y$ be compact Hausdorff spaces, $Z$ be the product space of $X$ and $Y$, and let $h \in C(Z \times Z)$ and $f \in C(Z)$ be given. The module of continuity of $f$ with respect to $h$ is a function $w_{h}(f):[0, \infty) \rightarrow \mathbb{R}$ defined by $w(f)(0)=0$, and

$$
w_{h}(f)(\delta)=\sup \{|f(u, v)-f(x, y)|:(u, v),(x, y) \in Z \text { and }|h((u, v),(x, y))|<\delta\}
$$

○2014 Uygun; licensee Springer. This is an Open Access article distributed under the terms of the Creative Commons Attribution License (http://creativecommons.org/licenses/by/2.0), which permits unrestricted use, distribution, and reproduction in any medium, provided the original work is properly cited. 
whenever $\delta>0$, with the following additional properties:

(i) $w(f)$ is increasing;

(ii) $\lim _{\delta \rightarrow 0}=0$.

We note that the above definition is motivated from [2, p.729] and generalizes the definition which is given there.

Definition 1.2 Let $X, Y$, and $Z$ be as in Definition 1.1. Let $h \in C(Z \times Z)$ be given. We define $H_{w, h}$ as the set of all continuous functions $f \in C(X \times Y)$ such that for all $(u, v),(x, y) \in X \times Y$, one has

$$
|f(u, v)-f(x, y)| \leq w_{h}(f)(|h((u, v),(x, y))|) .
$$

When $H_{w, h}$ is mentioned, we always suppose that $h$ satisfies the property for $H_{w, h}$ being a vector subspace of $C(X \times X)$. We note that $H_{w, h}$ has been considered in [2] by taking $X=[0, A], Y=[0, B](A, B>0)$,

$$
h((u, v),(x, y))=\left\|\left(f_{1}(u, v), f_{2}(u, v)\right)-\left(f_{1}(x, y), f_{2}(x, y)\right)\right\|,
$$

where

$$
f_{1}(u, v)=\frac{u}{1-u} \quad \text { and } \quad f_{2}(u, v)=\frac{v}{1-v} .
$$

The main result of this paper will be obtained via the following lemma.

\section{Main result}

Lemma 2.1 Let $X$ and $Y$ be compact Hausdorff spaces and $Z$ be a product space of $X$ and $Y$. Let $f_{1}, f_{2} \in C(Z)$ and $h \in C(Z \times Z)$ be defined by

$$
h((u, v),(x, y))=\left|\left(f_{1}(u, v), f_{2}(u, v)\right)-\left(f_{1}(x, y), f_{2}(x, y)\right)\right|
$$

so that $H_{w, h}$ is a subspace $C(X \times Y)$ and $f_{1}, f_{2} \in H_{w, h}(Z)$. Let $A: H_{w, h} \rightarrow C(Z)$ be a positive linear map. Let $(u, v) \in Z$ be given, and define $\varphi_{u, v}, \Phi_{u, v} \in C(Z)$ by

$$
\varphi_{u, v}=\left(f_{1}(u, v) f_{0}-f_{1}\right)^{2} \text { and } \Phi_{u, v}=\left(f_{2}(u, v) f_{0}-f_{2}\right)^{2} .
$$

Then, for all $(u, v) \in Z$, one has

$$
\begin{aligned}
0 & \leq A\left(\varphi_{u, v}+\Phi_{u, v}\right) \\
& \leq C_{1}\left[A\left(f_{0}\right)-f_{0}\right](u, v)-C_{2}\left[A\left(f_{1}+f_{2}\right)-\left(f_{1}+f_{2}\right)\right]+\left[A\left(f_{1}^{2}+f_{2}^{2}\right)-\left(f_{1}^{2}+f_{2}^{2}\right)\right],
\end{aligned}
$$

where

$$
C_{1}=\left(f_{1}(u, v)^{2}+f_{2}(u, v)^{2}\right) \text { and } C_{2}=-2\left(f_{1}(u, v)+f_{2}(u, v)\right) \text {. }
$$

Proof Note that

$$
0 \leq \varphi_{u, v}=f_{1}(u, v)^{2} f_{0}-2 f_{1}(u, v) f_{1}+f_{1}^{2} .
$$


Applying the linearity and positivity of $A$, we have

$$
0 \leq A\left(\varphi_{u, v}\right)=f_{1}(u, v)^{2} A\left(f_{0}\right)-2 f_{1}(u, v) A\left(f_{1}\right)+A\left(f_{1}^{2}\right) .
$$

Then one can have

$$
\begin{aligned}
0 \leq & A\left(\varphi_{u, v}\right)(u, v) \\
= & f_{1}(u, v)^{2} A\left(f_{0}\right)(u, v)-2 f_{1}(u, v) A\left(f_{1}\right)(u, v)+A\left(f_{1}^{2}\right)(u, v) \\
= & f_{1}^{2}(u, v)\left[A\left(f_{0}\right)(u, v)-f_{0}(u, v)+f_{0}(u, v)\right] \\
& -2 f_{1}(u, v)\left[A\left(f_{1}\right)(u, v)-f_{1}(u, v)+f_{1}(u, v)\right] \\
& +\left[A\left(f_{1}^{2}\right)(u, v)-f_{1}(u, v)^{2}+f_{1}(u, v)^{2}\right] \\
= & f_{1}^{2}(u, v)\left[A\left(f_{0}\right)-f_{0}\right](u, v)-2 f_{1}(u, v)\left[A\left(f_{1}\right)-f_{1}\right](u, v)+\left[A\left(f_{1}^{2}\right)-f_{1}^{2}\right](u, v) .
\end{aligned}
$$

Similarly, we have

$$
\begin{aligned}
A\left(\Phi_{u, v}\right)(u, v)= & f_{2}^{2}(u, v)\left[A\left(f_{0}\right)-f_{0}\right](u, v) \\
& -2 f_{2}(u, v)\left[A\left(f_{2}\right)-f_{2}\right](u, v)+\left[A\left(f_{2}^{2}\right)-f_{2}^{2}\right](u, v) .
\end{aligned}
$$

Now applying $A$, which is linear, to $\varphi_{u, v}+\Phi_{u, v}$ completes the proof.

Lemma 2.2 Let $X$ and $Y$ be compact Hausdorff spaces and $f_{1}, f_{2}$, and $h$ be defined as in Lemma 2.1. Let $f \in H_{w, h}$ be given. For each $\epsilon>0$, there exists $\delta>0$ such that

$$
|f(u, v)-f(x, y)|<\epsilon+\frac{2\|f\|}{\delta^{2}} h^{2}((u, v),(x, y)) .
$$

Proof Let $\epsilon>0$ be given. Since $w(f):[0, \infty) \rightarrow \mathbb{R}$ is continuous, there exists $\delta>0$ such that $w\left(f, \delta^{\prime}\right)=w(f)\left(\delta^{\prime}\right)<\epsilon$ for all $0 \leq \delta^{\prime}<\delta$. This implies, since

$$
|f(u, v)-f(x, y)| \leq w(f,|h((u, v),(x, y))|) \quad \text { for all }(u, v),(x, y) \in Z \text {, }
$$

that

$$
\left[\left(\varphi_{u, v}+\Phi_{u, v}\right)\right]^{\frac{1}{2}}(x, y)=|h((u, v)-h(x, y))|<\delta \text { implies }|f(u, v)-f(x, y)|<\epsilon,
$$

where $\varphi_{u, v}$ and $\Phi_{u, v}$ are defined as in Lemma 2.1. If $\left[\left(\varphi_{u, v}+\Phi_{u, v}\right)\right]^{\frac{1}{2}}(x, y) \geq \delta$, then

$$
|f(u, v)-f(x, y)| \leq 2\|f\| \leq 2\|f\| \frac{\left[\left(\varphi_{u, v}+\Phi_{u, v}\right)\right](x, y)}{\delta^{2}} .
$$

Hence, for all $(u, v) \in Z$, we have

$$
|f(u, v)-f| \leq \epsilon+2\|f\| \frac{\left[\left(\varphi_{u, v}+\Phi_{u, v}\right)\right]}{\delta^{2}} .
$$

This completes the proof. 
Lemma 2.3 Suppose that the hypotheses of Lemma 2.2 are satisfied. Let $f \in H_{w, h}$ and $\epsilon>0$ be given. Then there exists $C>0$ such that

$$
\|A(f)-f\|<\epsilon+C\left(\left\|A\left(f_{0}\right)-f_{0}\right\|+\left\|A\left(f_{1}+f_{2}\right)-\left(f_{1}+f_{2}\right)\right\|+\left\|A\left(f_{1}^{2}+f_{2}^{2}\right)-\left(f_{1}^{2}+f_{2}^{2}\right)\right\|\right) .
$$

Proof Set $K:=\frac{2\|f\|}{\delta^{2}}$. From Lemma 2.2, there exists $\delta>0$ such that for each $(u, v) \in Z$ we have

$$
\begin{aligned}
\left|f(u, v) f_{0}-f\right| & \leq \epsilon+\frac{2\|f\|}{\delta^{2}}\left[\varphi_{u, v}+\Phi_{u, v}\right] \\
& \leq \epsilon+\frac{2\|f\|}{\delta^{2}}\left[f_{1}^{2}(u, v) f_{0}+f_{2}^{2}(u, v) f_{0}-2 f_{1}(u, v) f_{1}-2 f_{2}(u, v) f_{2}+\left(f_{1}^{2}+f_{2}^{2}\right)\right],
\end{aligned}
$$

whence

$$
\begin{aligned}
\left|\left[A(f)-f(u, v) A\left(f_{0}\right)\right](u, v)\right| & \leq \epsilon A\left(f_{0}\right)(u, v)+K\left(A\left(\varphi_{u, v}\right)+A\left(\Phi_{u, v}\right)\right) \\
& =\epsilon+\epsilon\left[A\left(f_{0}\right)-f_{0}\right](u, v)+K A\left(\varphi_{u, v}+\Phi_{u, v}\right) .
\end{aligned}
$$

In particular, we have

$$
\begin{aligned}
|A(f)-f|(u, v) & \leq\left|\left[A(f)-f(u, v) A\left(f_{0}\right)\right](u, v)\right|+|f(u, v)|\left|\left(A\left(f_{0}\right)-f_{0}\right)(u, v)\right| \\
& \leq \epsilon+K A\left(\varphi_{u, v}+\Phi_{u, v}\right)(u, v)+(\|f\|+\epsilon)\left\|A\left(f_{0}\right)-f_{0}\right\| .
\end{aligned}
$$

Now, applying Lemma 2.1 and taking

$$
C=2 K+\|f\|,
$$

we have what is to be shown.

We note that in the above theorem $C$ depends only on $\|f\|$ and $\epsilon$, and is independent of the positive linear operator $A$.

We are now in a position to state the main result of the paper.

Theorem 2.4 Let $X$ and $Y$ be compact Hausdorff spaces and $Z$ be the product space of $X$ and $Y$. Let $f_{1}, f_{2} \in C(Z)$, and $h \in C(Z \times Z)$ be defined by

$$
h((u, v),(x, y))=\left\|\left(f_{1}(u, v), f_{2}(u, v)\right)-\left(f_{1}(x, y), f_{2}(x, y)\right)\right\|
$$

so that $H_{w, h}$ is a subspace $C(X \times Y)$ and $f_{1}, f_{2} \in H_{w, h}(Z)$. Let $\left(A_{n}\right)_{n \in \mathbb{N}}$ be a sequence of positive operators from $H_{w, h}$ into $C(X \times Y)$ satisfying:

(i) $\left\|A_{n}\left(f_{0}\right)-f_{0}\right\| \rightarrow 0$;

(ii) $\left\|A_{n}\left(f_{1}\right)-f_{1}\right\| \rightarrow 0$;

(iii) $\left\|A_{n}\left(f_{2}\right)-f_{2}\right\| \rightarrow 0$;

(iv) $\left\|A_{n}\left(f_{1}^{2}+f_{2}^{2}\right)-\left(f_{1}^{2}+f_{2}^{2}\right)\right\| \rightarrow 0$.

Then, for all $f \in H_{w, h}$, we have

$$
\left\|A_{n}(f)-f\right\| \rightarrow 0
$$


Proof Let $f \in H_{w, h}$ and $\epsilon>0$ be given. By Lemma 2.3, there exists $C>0$ (depending only on $\|f\|$ and $\epsilon>0$ ) such that for each $n$,

$$
\left\|A_{n}(f)-f\right\| \leq \epsilon+C\left(\left\|A_{n}\left(f_{0}\right)-f_{0}\right\|+\left\|A_{n}\left(f_{1}+f_{2}\right)-\left(f_{1}+f_{2}\right)\right\|+\left\|A_{n}\left(f_{1}^{2}+f_{2}^{2}\right)-\left(f_{1}^{2}+f_{2}^{2}\right)\right\|\right) .
$$

Since $\epsilon>0$ is arbitrary and the last three terms of the inequality converge to zero by the assumption, we have

$$
A_{n}(f) \rightarrow f
$$

This completes the proof.

Note also that in Theorem 1 of [2] it is not necessary to take a double sequence of positive operators: as the above result reveals, one can take $\left(A_{n}\right)$ instead of $\left(A_{n, m}\right)$.

\section{Remarks}

(1) If $X=[0,1]$, and $Y=\{y\}$ and $f_{1}, f_{2} \in C(X \times Y)$ are defined by

$$
f_{u, v}=u \text { and } f_{2}=0
$$

then Theorem 2.4 becomes the classical Korovkin theorem.

(2) If one takes $X=[0, A], Y=[0, B](0<A, B<1)$, and $f_{1}$ and $f_{2}$ are defined by

$$
f_{1}(u, v)=\frac{u}{1-u} \quad \text { and } \quad f_{2}(u, v)=\frac{v}{1-v}
$$

then the above theorem becomes Theorem 1 of [2].

(3) For linear positive operators of two variables, Theorem 2.4 generalizes the result of Volkov in [5].

(4) We believe that the above theorem can be generalized to $n$-fold copies by taking $Z=X_{1} \times X_{2} \times \cdots \times X_{n}$ instead of $Z=X \times Y$, where $X_{1}, X_{2}, \ldots, X_{n}$ are compact Hausdorff spaces.

(5) The above theorem is also true if one replaces $C(X)$ by $C_{b}(X)$, the space of bounded continuous functions, in the case of an arbitrary topological space $X$.

\section{Competing interests}

The author declares that they have no competing interests.

Received: 3 September 2013 Accepted: 5 February 2014 Published: 20 Feb 2014

\section{References}

1. Korovkin, PP: Linear Operators and Approximation Theory. Hindustan Publish Co., Delhi (1960)

2. Taşdelen, F, Erençin, A: The generalization of bivariate MKZ operators by multiple generating functions. J. Math. Anal. Appl. 331(1), 727-735 (2007)

3. Altomare, F, Campiti, M: Korovkin-Type Approximation Theory and Its Applications. de Gruyter, Berlin (1994)

4. Lorentz, GG: Approximation of Functions, 2nd edn. Chelsea, New York (1986)

5. Volkov, VI: On the convergence of sequences of linear positive operators in the space of continuous functions of two variables. Dokl. Akad. Nauk SSSR 115, 17-19 (1957) (in Russian) 